\title{
An efficient papaya leaf distortion mosaic potyvirus vector for virus-induced gene silencing in papaya
}

\author{
Decai Tuo ${ }^{1,2}$, Pu Yan ${ }^{1,2}$, Guangyuan Zhao ${ }^{1}$, Hongguang Cui ${ }^{3}$, Guopeng Zhu ${ }^{4}$, Yang Liu ${ }^{1,4}$, Xiukun Yang ${ }^{1,4}$, He Wang ${ }^{1,4}$, \\ Xiaoying $\mathrm{Li}^{1,2}$, Wentao Shen ${ }^{1,2,4,5}$ and Peng Zhou ${ }^{1,2,45}$
}

\begin{abstract}
Papaya (Carica papaya L.) is regarded as an excellent model for genomic studies of tropical trees because of its short generation time and its small genome that has been sequenced. However, functional genomic studies in papaya depend on laborious genetic transformations because no rapid tools exist for this species. Here, we developed a highly efficient virus-induced gene silencing (VIGS) vector for use in papaya by modifying an artificially attenuated infectious clone of papaya leaf distortion mosaic virus (PLDMV; genus: Potyvirus), PLDMV-E, into a stable Nimble Cloning (NC)-based PLDMV vector, PPLDMV-NC, in Escherichia coli. The target fragments for gene silencing can easily be cloned into PPLDMV-NC without multiple digestion and ligation steps. Using this PLDMV VIGS system, we silenced and characterized five endogenous genes in papaya, including two common VIGS marker genes, namely, phytoene desaturase, Mg-chelatase H subunit, putative GIBBERELLIN (GA)-INSENSITIVE DWARF1A and IB encoding GA receptors; and the cytochrome P450 gene CYP83B1, which encodes a key enzyme involved in benzylglucosinolate biosynthesis. The results demonstrate that our newly developed PLDMV VIGS vector is a rapid and convenient tool for functional genomic studies in papaya.
\end{abstract}

\section{Introduction}

Papaya (Carica papaya L., Caricaceae) is an economically important fruit tree cultivated in tropical and subtropical regions. ${ }^{1}$. Its flavorful fruit has the highest nutritional value among 38 common fruits because of its high concentrations of vitamins $\mathrm{A}$ and $\mathrm{C}$, potassium, folate, niacin, thiamine, riboflavin, iron, calcium, and fiber $^{2-4}$. In addition, in many tropical countries, other parts of the papaya plant, including seeds, leaves, roots, and flowers, are valued for their use in traditional medicine $^{4}$. Moreover, unripe (green) papaya fruit produces a

\footnotetext{
Correspondence: Wentao Shen (shenwentao@itbb.org.cn) or Peng Zhou (zhoupeng@itbb.org.cn)

${ }^{1}$ Key Laboratory of Biology and Genetic Resources of Tropical Crops, Ministry of Agriculture and Rural Affairs \& Institute of Tropical Bioscience and Biotechnology, Chinese Academy of Tropical Agricultural Sciences, 571101 Haikou, China

${ }^{2}$ Hainan Key Laboratory for Protection and Utilization of Tropical Bioresources \& Institute for Tropical Agricultural Resources, Chinese Academy of Tropical Agricultural Sciences, 571101 Haikou, China
}

Full list of author information is available at the end of the article latex that is the main source of papain, a proteolytic enzyme widely used in food processing, cosmetics, and medicine $^{5}$. In 2018, the Food and Agriculture Organization (FAO) estimated that the papaya cultivation area worldwide amounted to nearly 1.02 million hectares, with a total yield of 13.3 million metric tons of fruit.

Papaya is regarded as an excellent model system for genomic studies of both tropical trees and fruit trees because of its small diploid genome of 372 megabases $\mathrm{Mb})$ with nine pairs of chromosomes, its short generation time of 9-15 months, and its continual year-long flower and fruit production ${ }^{2,6}$. In 2008, a draft genome sequence of "SunUp" papaya, the first transgenic commercial papaya ringspot virus (PRSV)-resistant fruit crop, was obtained using a whole-genome shotgun approach and represented the fifth complete genome sequence of a flowering plant after Arabidopsis thaliana, Oryza sativa (rice), Populus trichocarpa (poplar), and Vitis vinifera

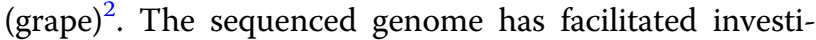
gations of the functions of many relatively poorly studied

\section{(c) The Author(s) 2021}

\footnotetext{
(c) Open Access This article is licensed under a Creative Commons Attribution 4.0 International License, which permits use, sharing, adaptation, distribution and reproduction cc) in any medium or format, as long as you give appropriate credit to the original author(s) and the source, provide a link to the Creative Commons license, and indicate if changes were made. The images or other third party material in this article are included in the article's Creative Commons license, unless indicated otherwise in a credit line to the material. If material is not included in the article's Creative Commons license and your intended use is not permitted by statutory regulation or exceeds the permitted use, you will need to obtain permission directly from the copyright holder. To view a copy of this license, visit http://creativecommons.org/licenses/by/4.0/.
} 
papaya genes. Moreover, recent rapid developments in next-generation sequencing technologies have enabled the production of abundant transcriptomic data associated with papaya tissue development, metabolism, and responses to biotic and abiotic stresses ${ }^{7-10}$. However, even with this increasing amount of genetic information, the capacity to study papaya gene functions using either forward or reverse genetic approaches remains limited. Forward genetic approaches are constrained by the lack of mutant papaya lines, and reverse genetic approaches are limited by the laborious and time-consuming stable genetic transformations necessary to take advantage of available genetic tools, such as RNA interference and gene editing ${ }^{11,12}$. In addition, papaya transformation protocols are not applicable to all papaya genotypes ${ }^{13,14}$. To improve papaya functional genomic studies and papaya breeding, the development of more rapid genetic tools is necessary.

Virus-induced gene silencing (VIGS) is a posttranscriptional gene silencing-based technique and it has been used as both forward and reverse genetics to assess the functions of single or multiple genes in plants ${ }^{15,16}$. In VIGS, a viral genome is designed to carry and replicate an incomplete gene sequence from the host organism to induce transient silencing of the endogenous gene. One advantage of VIGS over other methods is that silenced phenotypes can usually be observed within 3-4 weeks without time-consuming transformations ${ }^{17}$. In past decades, $>50$ different DNA and RNA viruses and their viral satellites have been developed as VIGS vectors in various plants $^{18}$. Through VIGS, many gene functions have been elucidated, including those involved in organ development, secondary metabolism, and plant-pathogen interactions $^{18-21}$. Nevertheless, VIGS has not yet been applied to functional genomics in papaya.

Papaya leaf distortion mosaic virus (PLDMV) is a member of the genus Potyvirus (family: Potyviridae) with a signal-stranded, positive-sense genomic RNA. Its genome comprises 10,153 nucleotides (nt), which encode a $373.68-\mathrm{kDa}$ polyprotein that is processed by viral proteases into a structural coat protein $(\mathrm{CP})$ and nine nonstructural proteins (P1, HC-Pro, P3, 6K1, CI, 6K2, NIaVPg, NIa-Pro, and NIb) ${ }^{22}$. Additionally, a small polyprotein, pretty interesting Potyviridae ORF (PIPO), is translated from an open reading frame (ORF) residing within the P3-encoding sequences due to slippage of the viral RNA polymerase ${ }^{22,23}$.

A key step in developing a reverse genetic system from RNA viruses is the construction of full-length infectious cDNA clones. As with most potyviruses, the development of infectious PLDMV cDNA clones has been difficult due to the large size of the genomic $\mathrm{RNA}^{24}$. The traditional construction of infectious potyviral full-length cDNA clones involves multiple time-consuming and error-prone subcloning steps using restriction enzymes and ligases. In previous studies, we addressed these limitations by assembling multiple viral fragments of PLDMV into different plasmid vectors for the construction of several infectious, full-length cDNA clones using sequence- and ligation-independent methods such as In-Fusion HD cloning and Gibson assembly cloning ${ }^{24,25}$. However, potyviral genomes are frequently unstable in Escherichia coli (E. coli) cloning amplification systems due to toxicity $^{24,26}$. To overcome this problem, we constructed a fulllength infectious PLDMV cDNA clone under the control of the T7 promoter by inserting an intron from Phaseolus vulgaris into the P3-encoding sequence, as this was known to stabilize viral cDNA in $E$. coli ${ }^{24}$. However, preparation of the infectious clone for inoculation requires in vitro viral RNA transcription, which is timeconsuming and expensive. Therefore, to facilitate the direct introduction of infectious cDNA clones into plants by inoculation, we developed an infectious PLDMV cDNA clone compatible with Agrobacterium-mediated infection, driven by the $35 \mathrm{~S}$ promoter of cauliflower mosaic virus $(C a M V 35 S)^{25}$. We accomplished this via direct transformation of Agrobacterium tumefaciens with a viral genome construct ${ }^{25}$. This strategy bypassed the requirement for cloning the viral sequences into E. coli, avoiding the problems of instability and toxicity.

To our knowledge, no potyvirus-based VIGS vectors for functional genomics have been reported for plants. In a previous study, potyvirus potato virus A (PVA) was modified to express the green fluorescent protein (GFP), resulting in systemic silencing of the GFP transgene in Nicotiana benthamiana (line 16c ${ }^{27}$. Similarly, our previous results showed that the foreign GFP gene inserted into the PLDMV genome was also processed as a viral gene into siRNAs by components involved in RNA silencing ${ }^{28}$. In addition, we obtained three mild strains of the virus for cross-protection: PLDMV-E, PLDMV-I, and PLDMV-EI ${ }^{29}$. These strains have single or double mutations of Lys (K)-to-Glu (E) in the Lys-Ile-Thr-Cys (KITC) motif and Arg (R)-to-Ile (I) in the Phe-Arg-Asn-Lys (FRNK) motif of the HC-Pro from the severe PLDMV strain DF (PLDMV-DF) ${ }^{29}$. The potyviral HC-Pro KITC and FRNK domains are two major determinants of symptom expression in infected plants ${ }^{30-32}$.

Our prior work on PLDMV, as well as the previous study on Nicotiana, provide clear bases for further development of Potyvirus-based VIGS vectors. Therefore, in this study, we modified the mild PLDMV-E strain to create a VIGS vector. We used the PLDMV-VIGS vector to induce silencing of five endogenous genes in papaya, namely, phytoene desaturase (PDS), Mg-chelatase H subunit (ChlH), GIBBERELLIN(GA)-INSENSITIVE DWARF1A(GID1A) and $1 B(G I D 1 B)$, and the cytochrome P450 gene CYP83B1. Our results demonstrate that the PLDMV-VIGS vector provides 
a rapid and efficient alternative for functional genomic studies in papaya.

\section{Results}

Construction of a stable Nimble Cloning-based PLDMV vector in E. coli using an attenuated PLDMV-E strain

To modify pPLDMV-E to an E. coli VIGS vector and facilitate the rapid cloning of foreign sequences into the PLDMV-E genome, we simultaneously assembled intron 2 of the NiR gene of $P$. vulgaris ${ }^{24}$ and the Nimble Cloning (NC) frame sequence (adapter $1-S f i I-c c d B$ gene$S f i$-adapter 2$)^{33}$ into the $P 3$ gene and at the junction of $\mathrm{NIb} / \mathrm{CP}$, respectively, within the PLDMV-E genome using Gibson assembly. In total, the plasmid construct, pPLDMV-NC, consisted of five DNA fragments: intron 2 (fragment II), the NC frame (fragment IV), two viral fragments (fragments I and III), and the backbone sequence of the pGreenII-35S vector and PLDMV CP gene (fragment V) (Fig. 1). We transformed this plasmid into $E$. coli strain DB3.1 and observed 22 colonies. Sequencing of the pPLDMV-NC vector showed that a total of 13 colonies contained the correct insert size and orientation without mutations, irregular deletions, or rearrangements. Thus, these pPLDMV-NC vectors could be used to clone target fragments flanked with the sequences of adapters 1 and 2 using the NC strategy (Fig. 1).

To test the infectivity of pPLDMV-NC after insertion of foreign genes, we cloned a 372-bp fragment of the GFP gene $(\triangle G F P)$ into pPLDMV-NC to generate pPLDMV- $\triangle$ GFP. The plants agroinoculated with PLDMV- $\triangle$ GFP began exhibiting mild mosaic symptoms 20 days post inoculation (dpi). The mild symptoms persisted until $60 \mathrm{dpi}$ and were similar to those in plants inoculated with PLDMV-E, whereas wild-type PLDMVDF caused severe mosaic symptoms, mottling, and distortion of the leaves (Fig. 2a). The results of the double-antibody sandwich-enzyme-linked immunosorbent assay (DAS-ELISA) showed that the accumulation of PLDMV- $\triangle$ GFP in systemically infected leaves was indistinguishable from the level of PLDMV-E but lower than that of PLDMV-DF (Fig. 2b). Thus, insertion of $\triangle G F P$ into pPLDMV-NC maintained similar infectivity and produced similar symptoms as those of PLDMV-E-infected plants. Therefore, PLDMV- $\triangle$ GFP was used as a nontarget control in further PLDMVbased VIGS assays.

\section{Silencing of two VIGS marker genes in papaya using pPLDMV-NC}

To test whether the pPLDMV-NC vector could be used to effectively induce endogenous gene silencing in papaya, we cloned a 348-bp fragment of papaya $P D S(P a P D S)$ and a 351-bp fragment of papaya $\mathrm{ChlH}(\mathrm{PaChlH})$ after genome-wide off-target gene silencing assessment into the pPLDMV-NC vector in sense orientation to generate pPLDMV-PaPDS and pPLDMV-PaChlH, respectively. The papaya seedlings agroinfiltrated with PLDMV-PaPDS and PLDMV-PaChlH initially developed mild photobleaching and a yellow-leaf phenotype along the veins at 15-20 dpi (Supplemental Fig. S1); more severe silencing phenotypes with large areas of photobleaching or yellowing in all newly developed leaves and stems were observed at 30-35 dpi (Fig. 3a). In contrast, leaves infected with PLDMV- $\Delta$ GFP showed typical mild mosaic symptoms (Fig. 3a). Reverse transcription-quantitative real-time PCR (RT-qPCR) showed that the PaPDS and $\mathrm{PaChlH}$ transcript levels in the corresponding leaves in which silenced phenotypes (SP1-3 and SH1-3) were observed at 30 dpi (Fig. 3b) were significantly reduced compared with those in PLDMV- $\triangle$ GFP-infected plants (Fig. 3c). These results suggest that the pPLDMV-NC vector can be used to silence endogenous genes in papaya.

To investigate the stability of the 348-bp PDS fragment in PLDMV-PaPDS, we analyzed the first (L1), second (L2), and third (L3) emerging leaves that maintained the photobleached phenotype at 50 and $60 \mathrm{dpi}$ (Fig. 4a) by RT-PCR using the primers pldmv8879F and pldmv9276R, which flanked the insert. The PaPDS insertion was stable in leaves L1 and L2 at 50 and 60 dpi but partially lost in L3 at 60 dpi. This suggests that the efficient silencing induced by the pPLDMV-NC VIGS vector was stable for $\sim 2$ months (Fig. 4b).

\section{Silencing of GID1 genes affected the growth and development of papaya plants}

To further assess the ability of the pPLDMV-NC VIGS vector to silence endogenous genes in papaya, we investigated the roles of two putative papaya GID1A (PaGID1A) and GID1B (PaGID1B) in the development of papaya plants. Following a genome-wide off-target gene silencing assessment, we chose two 273-bp, nonconserved fragments of PaGID1A and PaGID1B sharing 64\% nucleotide sequence identity to construct the pPLDMVPaGID1A and pPLDMV-PaGID1B silencing vectors using NC. Compared with the plants agroinfiltrated with PLDMV- $\triangle$ GFP, papaya plants infected with PLDMVPaGID1A or PLDMV-PaGID1B showed obvious phenotypic changes at $45 \mathrm{dpi}$. The stems of PLDMV-PaGID1Ainfected plants were slightly shorter, and their leaves were longer, thinner, and a darker shade of green (Fig. 5a, b). In contrast, plants infected with PLDMV-PaGID1B had an obvious dwarf phenotype with deformed, darker green leaves (Fig. 5a, b). In three replicate experiments, the PaGID1A and PaGID1B inserts were tested by RT-PCR, and analyses using RT-qPCR confirmed that in the infected plants, $P a G I D 1 A$ and $P a G I D 1 B$ expression was significantly downregulated, although by different amounts (Supplemental Fig. S2). Together, silencing of 


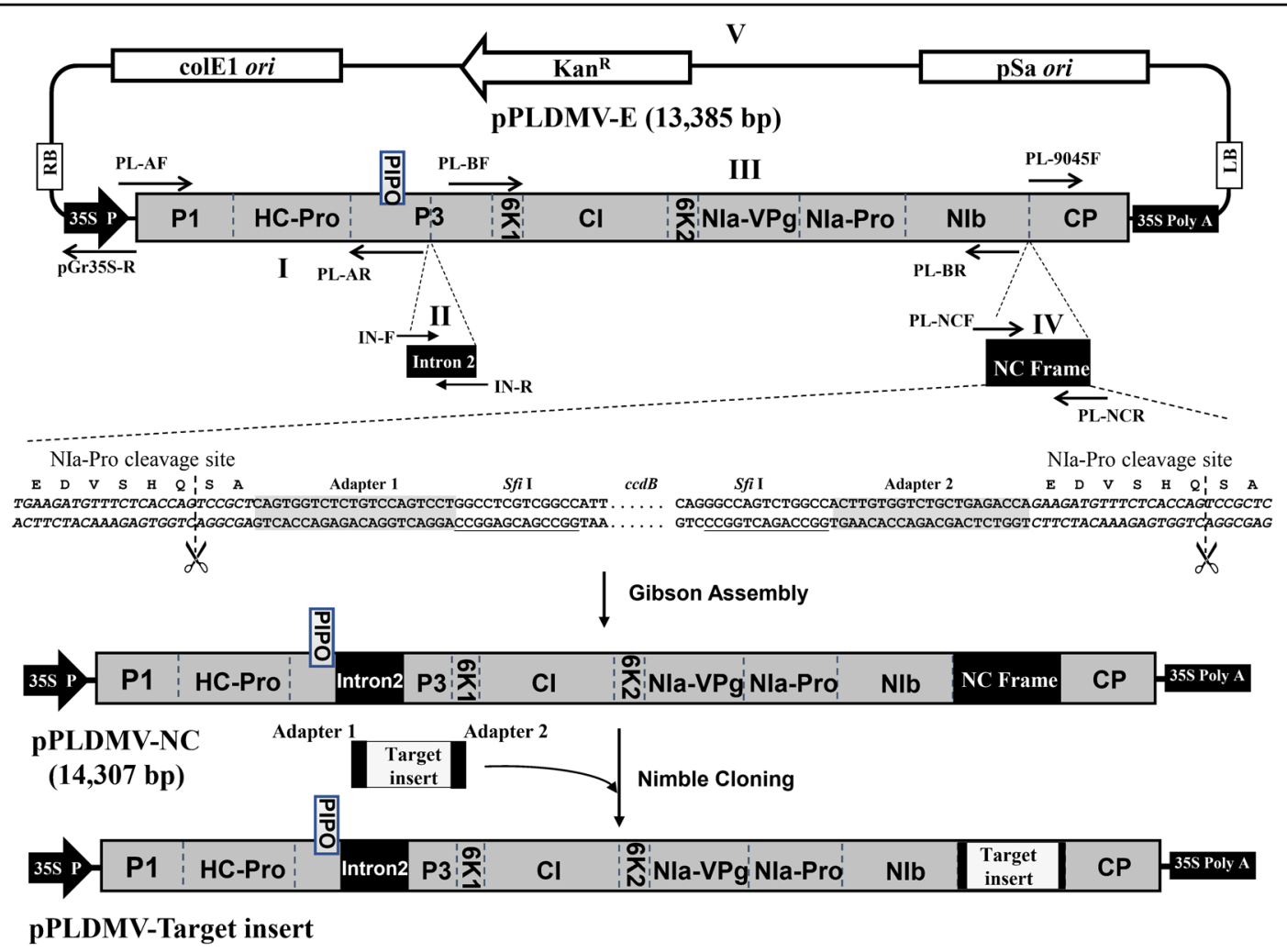

Fig. 1 Schematic representation of the PLDMV-NC vector. Construction of the PLDMV-NC vector. Intron 2 (II) of the NiR gene and the NC frame sequence (adapter 1-Sfil-ccdB gene-Sfil-adapter 2, IV) were individually assembled into the $P 3$ gene and at the junction of $\mathrm{N} / \mathrm{lb} / \mathrm{CP}$, respectively, within the PPLDMV-E vector via Gibson assembly along with two PLDMV fragments (I and III) and the fragment containing the backbone of pGreenII$35 S$ and PLDMV-CP $(V)$ to generate the pPLDMV-NC construct. Within the plasmid, the transformed portion comprises five sections (I-V). PLDMV-NC was fused between the CaMV35S promoter (CaMV35S) and poly(A) signal of the T-DNA binary vector pGreenll-35S. The target gene fragment was flanked by adapters 1 and 2 of the NC frame and could be cloned into the pPLDMV-NC vector using Nimble Cloning (NC). Eleven major open reading frames (ORFs) of the PLDMV genome are indicated by gray boxes: P1, HC-Pro, P3, PIPO, 6K1, Cl, 6K2, VPg, N/a-Pro, N/b, and CP. White rectangles and arrows indicate the elements of the pGreenll-35S vector backbone. The octapeptide recognized by the Nla protease is underlined, and its nucleotide sequence is shown in italics. Scissors indicate the Nla-Pro cleavage site. The nucleotide sequences of adapters 1 and 2 in the NC frame sequence are shaded. The Sfil sites are underlined, and the $c c d B$ gene is marked by dotted lines

$P a G I D 1 A$ or $P a G I D 1 B$ had dramatic effects on the growth and development of papaya plants.

\section{Silencing of CYP83B1 reduced papaya leaf benzylglucosinolate (BGLS) accumulation}

We further targeted papaya CYP83B1 (PaCYP83B1), a gene in the BGLS biosynthetic pathway ${ }^{34}$, using the pPLDMV-NC vector. After a genome-wide off-target gene silencing assessment, a 348-bp fragment of $\mathrm{PaCYP83B1}$ was inserted into the PPLDMV-NC vector in sense orientation to generate pPLDMV-PaCYP83B1, which was then transformed into $A$. tumefaciens for inoculation of the papaya plants. A lesion-mimicking phenotype was first observed at 20 dpi in newly developed leaves of plants infected with PLDMV-PaCYP83B1 (Fig. 6a), and the phenotype became more obvious by 45 dpi (Fig. 6a). High-performance liquid chromatography (HPLC) showed that in leaves infected with PLDMV-
PaCYP83B1, the BGLS concentration was significantly reduced compared with that in leaves of the control plants infected with PLDMV- $\triangle$ GFP (Fig. 6b, c). This corresponded with a significant decrease in $\mathrm{PaCYP} 83 \mathrm{~B} 1$ mRNA transcripts in PLDMV-PaCYP83B1-infected plants (Supplemental Fig. S3). These results suggest that PaCYP83B1 is a key enzyme in the papaya BGLS pathway.

\section{Discussion}

In this study, we report the development of papayainfecting potyvirus as a VIGS vector for RNA silencing of endogenous papaya genes. The PLDMV-based VIGS system provides an effective and valuable tool for functional genomics in papaya.

The severity of the symptoms due to viral infection could interfere with gene function analysis and VIGS phenotypic evaluation in plants. Therefore, we recently 
a

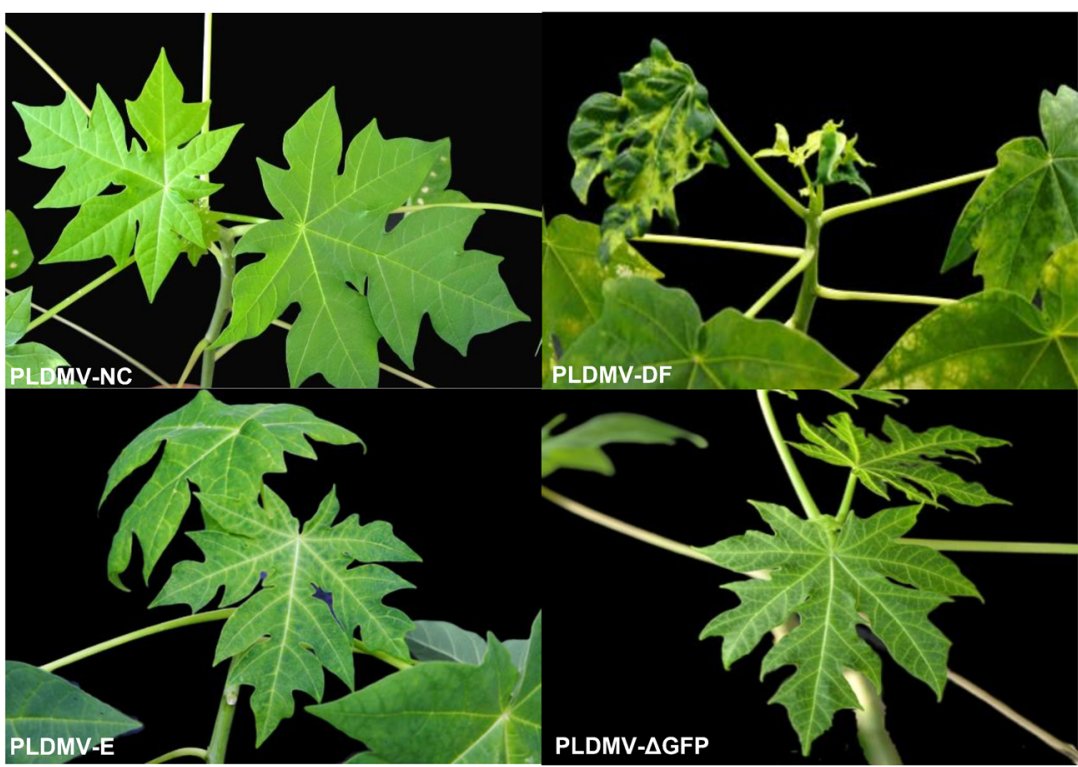

b

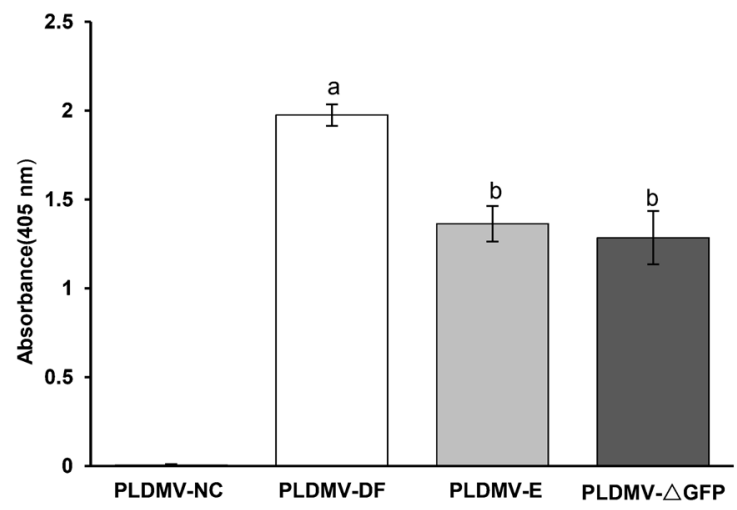

Fig. 2 Symptom development and infectivity levels of PLDMV- $\triangle$ GFP in papaya plants. a Systemic symptoms induced by wild-type (WT) PLDMV (PLDMV-DF), PLDMV-E, and PLDMV- $\triangle$ GFP in papaya plants at 60 days post inoculation (dpi). b Detection of PLDMV-DF, PLDMV-E, and PLDMV$\triangle$ GFP accumulation in infected papaya leaves at $60 \mathrm{dpi}$ using PLDMV coat protein-specific antiserum. Three independent experiments were performed, and each included five plants per treatment group. Error bars indicate SDs. Different letters indicate significant differences according to Duncan's multiple-range test $(P<0.05)$

engineered three mild strains of PLDMV (PLDMV-E, PLDMV-I, and PLDMV-EI) from the severe PLDMV-DF strain by single or double mutations of $\mathrm{K}$ to $\mathrm{E}$ in the KITC motif and R to I in the FRNK motif of the HCPro $^{29}$. However, PLDMV-I and PLDMV-EI showed a lower level of viral accumulation than PLDMV-E in systemically infected leaves. Because the lower levels of viral accumulation might not trigger effective VIGS, we selected attenuated PLDMV-E for development as a VIGS vector. Indeed, the resulting nontarget control based on PLDMV-E, PLDMV- $\triangle$ GFP, induced the same mild symptoms as PLDMV-E in papaya, which helped to distinguish between the phenotypes of target gene silencing and symptoms due to viral infection in the VIGS assay. In addition, previous studies revealed that the mutation of $\mathrm{K}$ to $\mathrm{E}$ in the HC-Pro KITC motif resulted in a loss of aphid transmission of a potyvirus ${ }^{30}$. Thus, this mutation might reduce the risk of escape of the PLDMV-E-based VIGS vector into the environment.

A major limiting factor in the development of VIGS vectors from potyviruses is the inefficiency of the cloning and manipulation of potyviral genomes because they consist of one large RNA molecule of $\sim 10,000$ nt that encodes a single polyprotein, which frequently makes the clone unstable in bacterial cells ${ }^{24,26}$. In this study, we introduced intron 2 from $P$. vulgaris NiR into the P3encoding sequence of PLDMV-E based on our previously successful efforts to modify PLDMV-E as a VIGS vector for stable propagation and easy manipulation in $E$. $\operatorname{coli}^{24}$. In addition, we inserted the NC frame into PLDMV-E to 
a
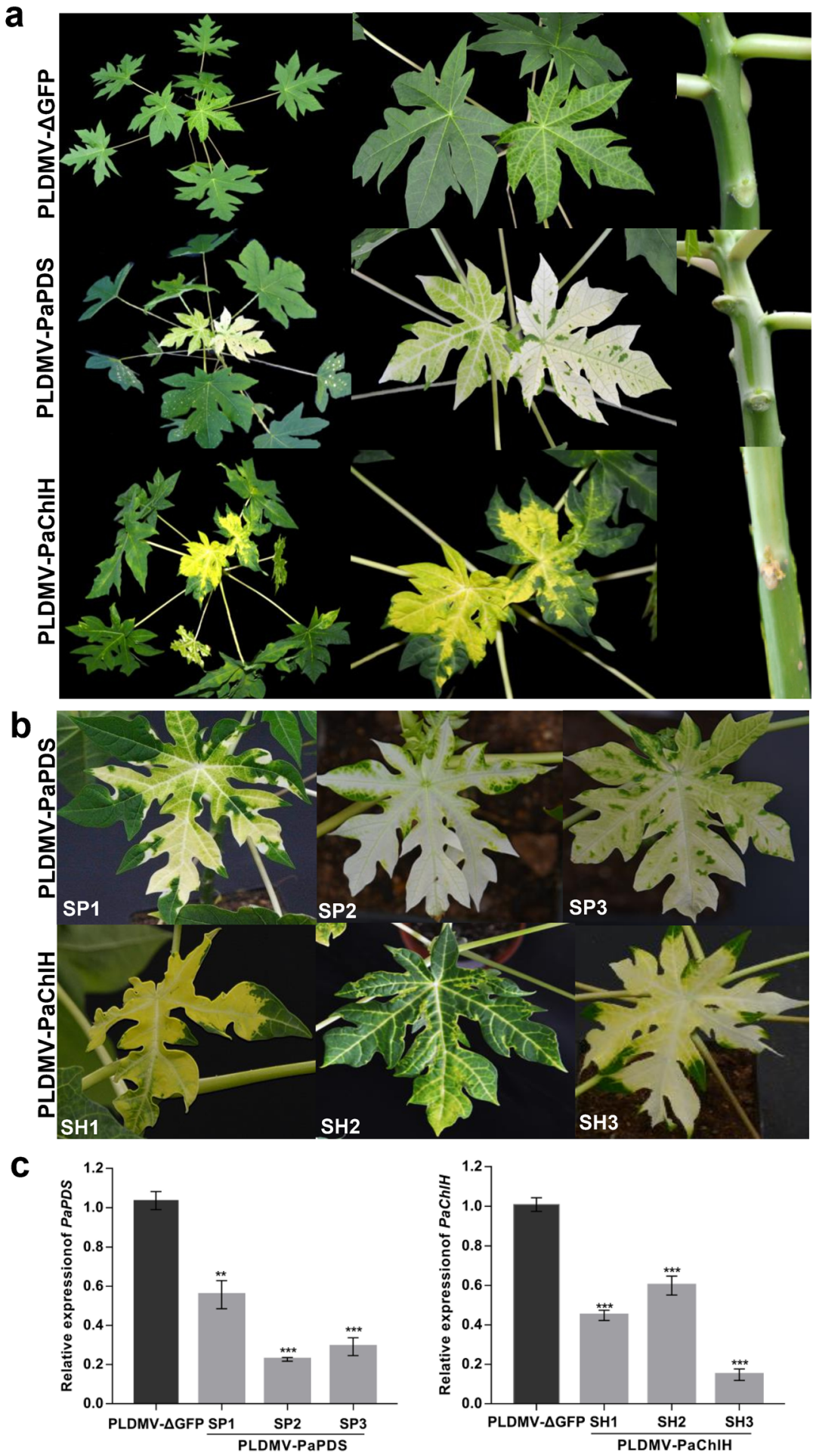

Fig. 3 Silencing of PaPDS and PaChIH genes in papaya using the PLDMV VIGS vector. a Papaya plants were agroinoculated with PLDMV-NC carrying a 348-bp fragment of PaPDS (PLDMV-PaPDS) or a 351-bp fragment of PaChIH (PLDMV-PaChlH). Compared with plants agroinoculated with the PLDMV- $\triangle$ GFP control, which showed typical mosaic virus symptoms, photobleaching and yellowing were observed in newly developed leaves and stems infected with PLDMV-PaPDS and PLDMV-PaChlH, respectively, at 30 days post inoculation (dpi). b The leaves (SP1-SP3 and SH1-SH3) developed albino phenotypes at $30 \mathrm{dpi}$, as expected for PaPDS and PaChIH silencing. c Relative expression of PaPDS and PaChIH determined by RT-qPCR in leaves (SP1-SP3 and SH1-SH3) exhibiting the silencing phenotype. Statistical analysis to compare the plants with those infected with the nontarget control PLDMV- $\triangle$ GFP was carried out by Student's $t$ test $\left({ }^{* *} P<0.01\right.$ and $\left.{ }^{* * *} P<0.001\right)$. Error bars indicate the SDs of three technical replicates for each individual sample, except for the control, the error bars for which represent the SDs of nine plants 


\section{a}
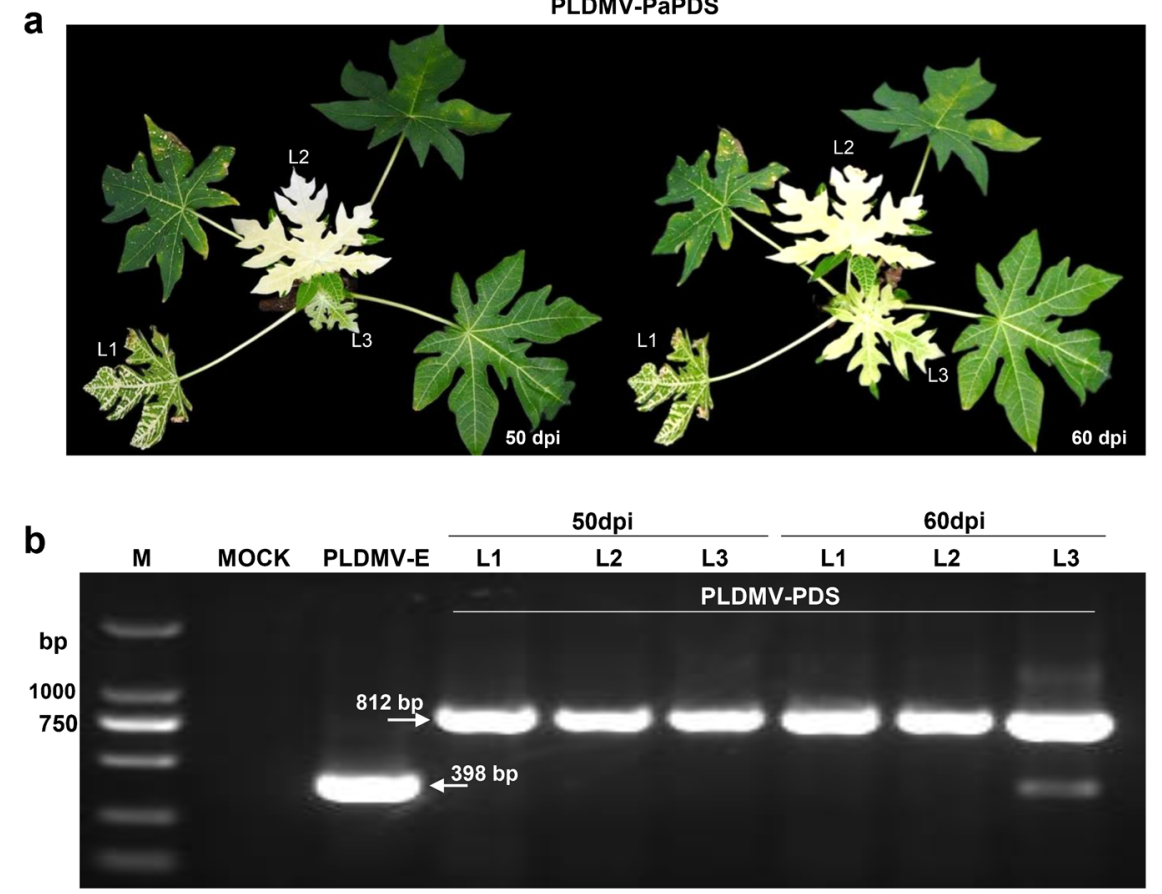

Fig. 4 Stability of the inserted PaPDS fragment in leaves systemically infected with PLDMV-PaPDS. a Papaya plants infected with PLDMVPaPDS at 50 and 60 days post inoculation (dpi). b The first, second, and third emergent leaves (L1-3, respectively) above the inoculated leaves were harvested at 50 and $60 \mathrm{dpi}$, and the PaPDS fragment was amplified by RT-PCR with primer pairs that flanked the insert to assess insert stability during systemic movement

facilitate the rapid cloning of desired gene fragments for silencing into PLDMV-NC. The resulting pPLDMV-NC vector containing intron 2 and the $\mathrm{NC}$ sequence was stable in E. coli. Target fragments flanked with NC adapters introduced by PCR can easily be cloned into pPLDMV-NC via a simple mixture of the rare-cutting restriction enzyme SfiI and T5 exonucleases, which simultaneously accomplish vector linearization and the assembly reaction in one tube ${ }^{33}$. In previous studies, Gateway-based and ligation-independent VIGS vectors have been used for rapidly cloning target fragments without the multiple steps of restriction digestion and ligation $^{15,35}$. In contrast to these approaches, NC does not require an additional VIGS vector linearization step; therefore, it is more cost-effective than Gateway-based and ligation-independent cloning methods ${ }^{33}$. In this study, $>90 \%$ of the clones were positive in each transformation using $\mathrm{NC}$ with the mixture of pPLDMV-NC vector and PCR amplicons representing individual target gene fragments.

Several strategies exist for inserting target fragments into the genomes of plant viruses without affecting viral infectivity. These include the substitution of unnecessary parts of viral ORFs, fusion of target fragments into an ORF, and expression of the target fragments as an extra gene by a duplicated or heterologous subgenomic viral promoter ${ }^{18,36}$. Potyvirus-based vectors usually employ fusion strategies. Specifically, target fragments may be fused into the ORF of a large polyprotein at the $\mathrm{N}$-terminus of $P 1$, between the $P 1$ and HC-Pro cistrons or between the NIb and CP cistrons $^{27,37,38}$. In this study, we inserted the NC frame between the NIb and CP cistrons of PLDMV-E to facilitate rapid cloning of the desired gene fragments for silencing. To enable infection by PLDMV-NC after a target fragment has been cloned into the NC frame, the inserted fragment should be translated as part of the potyviral polyprotein during infection, and the resultant expressed protein insertion should be released from the viral polyprotein precursor by original and additional duplication of NIa-Pro proteolytic $\operatorname{sites}^{25,38}$. Therefore, the base pairs of the target sequence cloned into an NC frame should be multiples of three and cannot contain any stop codons. In addition, the NC frame includes a relatively rare-cutting restriction enzyme, $S f i$. Therefore, the inserted target sequence cannot contain its recognition site. However, $S$ fiI restriction sites are rare in plant genomes ${ }^{33,39}$.

The PDS gene, involved in carotenoid biosynthesis, and the $\mathrm{ChlH}$ gene, involved in chlorophyll biosynthesis, have been widely used to generate visual phenotypes in plant VIGS experiments across many species ${ }^{18}$. Silencing PaPDS 


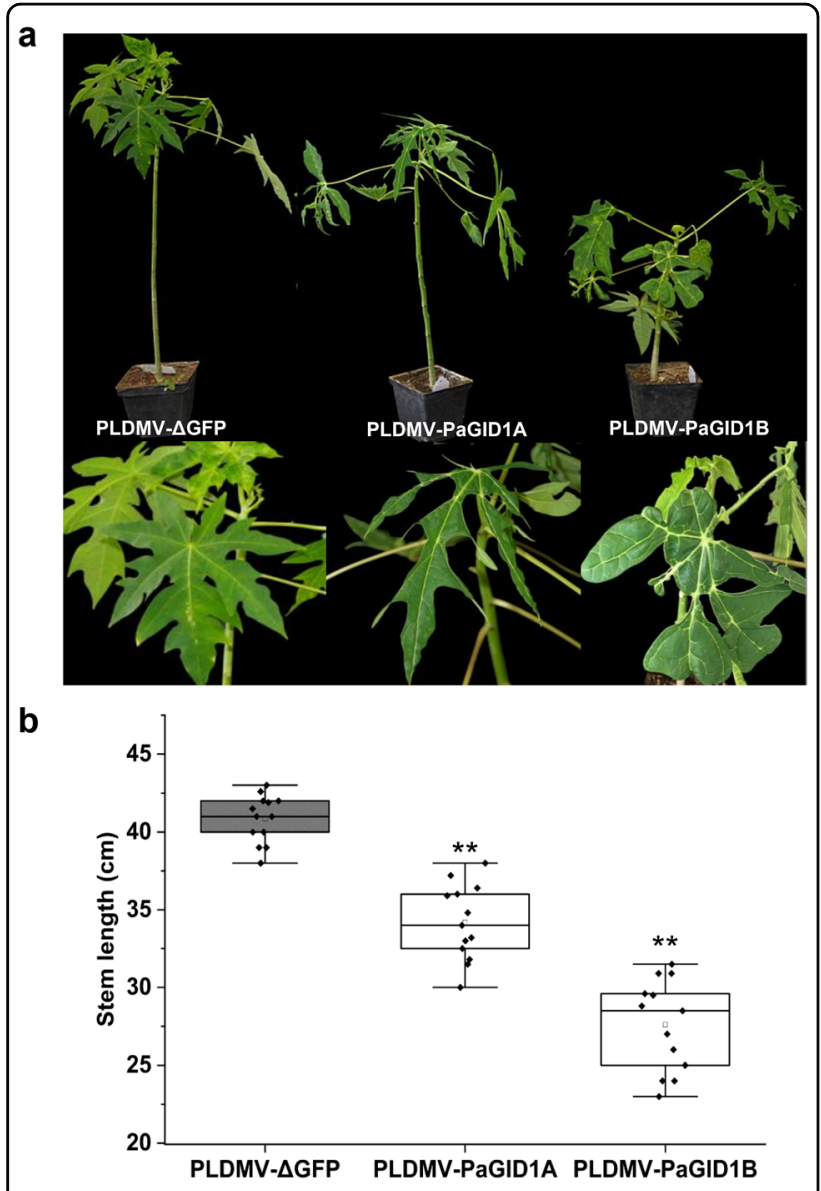

Fig. 5 Silencing of $P a G I D 1 A$ and $P a G I D 1 B$ in papaya plants. a Silencing of $P a G I D 1 A$ or $P a G I D 1 B$ resulted in a dwarf phenotype and affected the leaf morphology of papaya plants. $\mathbf{b}$ Stem lengths of papaya plants infected with PLDMV-PaGID1A and PLDMV-PaGID1B and the nontarget control PLDMV- $\triangle$ GFP at 45 days post inoculation (dpi). Three independent experiments were performed, and each included five plants per treatment group. Statistical tests to compare the plants with those infected with the nontarget control PLDMV$\triangle$ GFP were performed by Student's $t$ test ( $\left.{ }^{* *} P<0.01\right)$. Error bars indicate SDs

and $\mathrm{PaChlH}$ in papaya resulted in a photobleached or yellow-leaf phenotype, affecting the entire surface of newly developed leaves and stems, that persisted until at least 60 dpi. This indicates that the PLDMV-E-based VIGS system is highly efficient at silencing in papaya. Moreover, we investigated the stability of the target inserts from the PLDMV-E-based VIGS vector in leaves systemically infected with PLDMV-PaPDS. Deletions in the inserts were detectable in L3 emerging leaves by $60 \mathrm{dpi}$. Therefore, the long duration of silencing by this PLDMV-E-based VIGS system makes it a valuable tool for functional studies of genes involved in developmental and biosynthetic pathways and stress tolerance in papaya.

The development of dwarf fruit trees can increase fruit yield and facilitate production management ${ }^{40}$. The acquisition of dwarf traits represents a major objective in papaya breeding, and these traits are often related to GA, which are essential hormones for plant growth. The GA receptor GID1 plays a crucial role in the GA signaling pathway ${ }^{41,42}$. In this study, we cloned two GID1like genes encoding GA receptors, namely, PaGID1A and $P a G I D 1 B$, from the papaya genome. Silencing of $P a G I D 1 A$ using VIGS resulted in only slight dwarfing, whereas silencing of $P a G I D 1 B$ produced a severely dwarfed phenotype (Fig. 5a). Moreover, silencing of each of two receptor genes strongly impacted the morphology of papaya leaves. These results indicate that PLDMV-PaGID1A or PLDMV-PaGID1B infectioninduced typical GID1-silencing phenotypes similar to those observed by tobacco rattle virus-induced gene silencing of GID1s in petunia ${ }^{43}$ and for Arabidopsis and tomato gid1 mutants ${ }^{44,45}$.

Glucosinolates (GLSs) and their hydrolysis products are amino acid-derived secondary metabolites characteristic of the Brassicaceae family, with important roles in flavor formation, plant defense, and human cancer prevention $^{46,47}$. To date, $>30$ GLSs have been found in the model plant Arabidopsis ${ }^{48}$. In contrast, papaya tissues contain only one kind of GLS, BGLS ${ }^{48,49}$. Within the GLS pathway in Arabidopsis, CYP83B1 is the key enzyme involved in oxime metabolism ${ }^{50}$. In this study, silencing of PaCYP83B1 resulted in reduced BGLS accumulation, suggesting that PaCYP83B1 is one of the key enzymes of the papaya BGLS pathway. Moreover, PaCYP83B1silenced papaya plants displayed necrotic lesions on leaves, which is consistent with the phenotype of Arabidopsis cyp $83 B 1$ mutants $^{51}$. Lesion mimic formation in cyp $83 B 1$ mutants may be due to the reduction in GLS defense compounds and upregulation of other defense pathways, such as those involving methyl jasmonate and salicylic acid $^{51}$. In addition, the CYP83B1 enzyme has been identified as a metabolic branch point in auxin (IAA) and indole GLS biosynthesis in Arabidopsis ${ }^{52}$. Thus, cyp83B1 loss-of-function mutations contributed to adventitious root formation in Arabidopsis because indolic compounds were redirected to IAA synthesis when the cyp83B1 mutation blocked the synthesis of indole glucosinolate (indole GLS) ${ }^{51,52}$. However, we did not observe the adventitious root phenotype in PaCYP83B1-silenced papaya plants. This may be because papaya contains BGLS but not indole GLS; thus, the silencing of $\mathrm{PaCYP83B1}$ may not affect IAA synthesis in this species ${ }^{47,49}$.

In future studies, we will attempt to use this approach to determine the functions of genes involved in floral organ and fruit development and sex determination in papaya. In addition, PLDMV was found to infect some cucurbitaceous plants, such as Cucumis metuliferus, Cucurbita pepo, and Cucumis sativus. Therefore, this PLDMV-based 


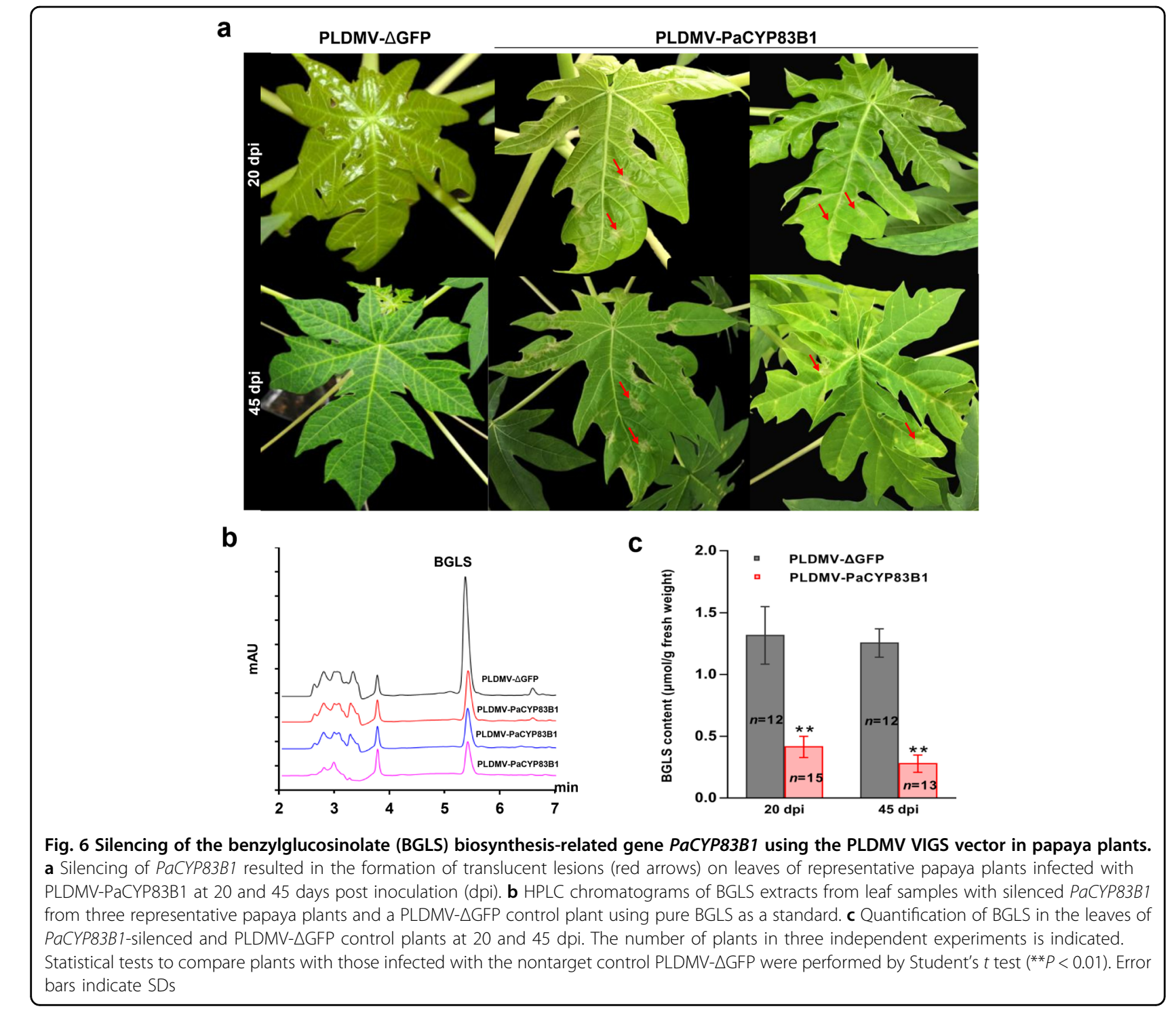

PLDMV-PaCYP83B1

VIGS vector will be used for functional genomic studies in cucurbits $^{53}$. In our study, the combination of the Gibson assembly and Nimble cloning methods made potyvirusbased vector construction and genetic engineering simpler and more effective than observed with restriction/ ligation-based cloning methods. Potyvirus is the largest plant-infecting RNA virus genus, with a wide host range, including both dicots and monocots ${ }^{54}$. Thus, our construction strategies are potentially applicable to other potyviruses that infect nonmodel species without wellestablished VIGS techniques and genetic transformations.

\section{Materials and methods}

\section{Plant materials}

"Mihong" papaya seedlings were used in this study. For the VIGS assays, the agroinfiltrated plants were grown in a greenhouse and maintained at $23^{\circ} \mathrm{C}$ under $16 / 8 \mathrm{~h}$ light/ dark photoperiods with a light intensity of $300 \mu \mathrm{mol} \mathrm{m}{ }^{-2}$ $\mathrm{s}^{-1}$ and $60 \%$ relative humidity.

\section{Plasmid construction}

Gibson assembly was used to insert intron 2 (220 bp) of the P. vulgaris NiR gene and the NC frame (adapter 1$S f i \mathrm{I}-c c d B$ gene-SfiI-adapter 2) into the PLDMV-E cDNA within the $P 3$ gene and at the junction of $N I b / C P$, respectively (Fig. 1). These insertions comprised five DNA fragments (I, II, III, IV, and V); the intron 2 represented fragment II, and the NC frame was fragment IV within the pPLDMV-NC construct. Intron 2 from the pT7-PLDMVIn2 vector and the $\mathrm{NC}$ frame from the $\mathrm{pNC}-\mathrm{UC}$ vector were amplified using the primer pairs IN-F/R and PLNCF/NCR, respectively ${ }^{24,33}$. The previously generated pPLDMV-E plasmid ${ }^{29}$ was used for amplification of fragments I, III, and $\mathrm{V}$ in PPLDMV-E, including the 
full-length viral genomic sequence and the backbone of the mini-binary promoter in pGreenII-35S using the primer pairs PL-AF/AR, PL-BF/PL-BR, and PL-9045F/ pGr35S-R, respectively. All primer pairs yielded sequences overlapping adjacent fragments by 15 and 25 bases and are listed in Supplementary Table 1. The Gibson assembly reaction was performed in a total volume of $10 \mu \mathrm{L}$, containing $5 \mu \mathrm{L}$ of $2 \times$ Gibson mix (NEB, Ipswich, MA, USA) and $100 \mathrm{ng}$ of each purified PCR fragment. The reaction mix was incubated at $50^{\circ} \mathrm{C}$ for $15 \mathrm{~min}$ and then placed on ice for transformation into E. coli DB3.1 competent cells. The E. coli colonies were screened for successful transformation with PCR using the primer pair PL-NCF/NCR (Supplementary Table 1); transformants positive for the PPLDMV-NC plasmids were confirmed by sequencing. NC was applied to the pPLDMV-NC constructs to obtain the inserts of interest. The GFP fragment (nts 1428-1775 of GFP; GenBank accession: MK896905) was amplified from the pPLDMV-GFP plasmid $^{38}$ and cloned into pPLDMV-NC to generate pPLDMV- $\triangle$ GFP as a nontarget control for VIGS.

For VIGS, the selected regions of target genes for genome-wide off-target gene silencing were checked using pssRNAit (http://plantgrn.noble.org/pssRNAit/). A 348-bp fragment of papaya PDS (nts 892-1239 of PaPDS; GenBank accession: DQ779922), a 351-bp fragment of papaya $\mathrm{ChlH}$ (nts 3991-4341 of PaChlH; GenBank accession: XM_022037628), 273-bp fragments of papaya GID1A and GID1B genes (nts 196-468 of PaGID1A and nts 766-1038 of PaGID1B; GenBank accessions: MT780505 and XM_022046316, respectively), and a 348-bp fragment of papaya CYP83B1 (nts 786-1133 of PaCYP83B1; GenBank accession: XM_022047103) were amplified using papaya cDNA as the template with the corresponding primer pairs (Supplementary Table 2). Then, the amplified fragments were cloned into pPLDMV-NC to generate pPLDMVPaPDS, pPLDMV-PaChlH, pPLDMV-PaGID1A, pPLDMVPaGID1B, and pPLDMV-PaCYP83B1. All NC reactions were carried out in $10-\mu \mathrm{L}$ reactions, containing $5 \mu \mathrm{L}$ of $2 \times$ Nimble Mix, $10-50$ ng of PCR insert, and DNase/RNasefree $\mathrm{dH}_{2} \mathrm{O}^{33}$. The reaction mixture was incubated in a water bath for $1 \mathrm{~h}$ at $50^{\circ} \mathrm{C}$ before being used to transform $E$. coli strain $\mathrm{DH} 5 \alpha$ via the heat-shock method. All resulting constructs were sequenced to verify the accuracy of insertions prior to the transformation of A. tumefaciens GV3101 with the pSoup helper plasmid.

\section{Agroinfiltration of papaya plants}

We grew a single colony of $A$. tumefaciens strain GV3101 overnight in Luria-Bertani medium containing rifampicin $(25 \mathrm{mg} / \mathrm{L})$ and kanamycin $(50 \mathrm{mg} / \mathrm{L})$ at $28^{\circ} \mathrm{C}$ with shaking for each pPLDMV-NC construct. Thereafter, the agrobacterial cells were harvested by centrifugation at $2500 \times \mathrm{g}$ and $4{ }^{\circ} \mathrm{C}$ for $5 \mathrm{~min}$ and then resuspended in infiltration buffer $(10 \mathrm{mM} \mathrm{MgCl}, 10 \mathrm{mM}$ 2-(N-morpholino) ethanesulfonic acid ( $\mathrm{pH}$ 5.5), and $100 \mu \mathrm{M}$ acetosyringone) to obtain an $\mathrm{OD}_{600}$ of 0.5 . The cells were stored at room temperature for 2 to $3 \mathrm{~h}$ in the dark. The backs of the leaves of 7- to 8-week-old papaya seedlings were inoculated with the transformed Agrobacterium using a 1-mL needleless syringe. We repeated each agroinfiltration experiment at least three times for each viral construct.

\section{RT-PCR, RT-qPCR, and DAS-ELISA}

The total RNA was extracted from $100 \mathrm{mg}$ of symptomatic papaya leaves using TRIzol reagent (Thermo Fisher Scientific, Waltham, MA, USA) and digested with DNase (Takara Bio Inc., Kusatsu, Shiga, Japan) to remove genomic DNA. For RT-PCR, first-strand cDNAs from $1.0 \mu \mathrm{g}$ of total RNA were synthesized using the Takara RNA PCR Kit (AMV) Ver. 3.0 (Takara Bio Inc.) with random nonamers and oligo(dT) adaptor primers. To test the stability of the inserted fragments in PLDMV-NC derivatives, RT-PCR was performed using the pldmv8879F (5'-G GAACAAGCACCATACAATCATCT-3') and pldmv9 276R (5'-GTGGTATAATGAAAGATCCGCTTGA-3') primers, which flank the $3^{\prime}$ end of $N I b$ and the $5^{\prime}$ end of $C P$, respectively. The cDNAs for RT-qPCR were prepared from $1 \mu \mathrm{g}$ of DNA-free RNA with oligo(dT) using the PrimeScript RT Reagent Kit (Takara Bio Inc.) following the manufacturer's instructions. All RT-qPCR reactions were carried out using the SYBR Premix Ex Taq II Kit (Takara Bio Inc.); the papaya actin gene (GenBank accession: AY906938) was used as an internal control ${ }^{29}$. The species-specific primer pairs for PaPDS, PaChlH, PaGID1A, PaGID1B, and PaCYP83B1 (Supplementary Table 3) were used to test the effectiveness of silencing for each of these genes, and target gene expression levels were calculated using the delta-delta Ct method and compared with their expression levels in the PLDMV-GFP-infected samples $^{29}$. The accumulation levels of PLDMV-DF, PLDMV-E, and PLDMV- $\triangle$ GFP in systemically infected plants were determined using DAS-ELISA with the antiserum to the PLDMV coat protein (CP) as described previously $^{29}$.

\section{Benzylglucosinolate (BGLS) extraction and HPLC analysis}

Glucosinolate extraction was performed as described previously ${ }^{55}$. Then, $10 \mu \mathrm{L}$ of the sample was injected onto a COSMOSIL 5C18-MS-II column (4.6 mm I.D. $\times$ $250 \mathrm{~mm}$ ) (Nacalai Tesque Inc., Kyoto, Kyoto, Japan) for HPLC analysis using a mobile phase of $0.01 \%$ trifluoroacetic (TFA) acid in water (v/v) (solvent A) and acetonitrile with $0.1 \%$ TFA $(\mathrm{v} / \mathrm{v})$ (solvent B) on an Agilent 1260 Infinity II LC System (Agilent, Santa Clara, CA, USA). The peak of BGLS was identified at $229 \mathrm{~nm}$ using pure BGLS from Sigma Aldrich as a standard. 
The amount of BGLS in each sample was quantified using the equation of the regression line: $y=2748.3 x+$ $253.43(y=$ peak area; $x=$ final concentration $(\mu \mathrm{g} / \mathrm{ml})$ of BGLS in the sample), with a correlation coefficient of $0.9965\left(R^{2}\right)$. Finally, we normalized the amount of BGLS in each sample per gram of fresh weight extracted. Three independent experiments were performed, and each included four to five plants per treatment group.

\section{Acknowledgements}

This work was supported by the Hainan Provincial Natural Science Foundation of China (2019RC298 and 2018CXTD343), the National Natural Science Foundation of China (32072390), and the Central Public Interest Scientific Institution Basal Research Fund for Chinese Academy of Tropical Agricultural Sciences (grant no. 19CXTD-33). We thank Fandong Kong and Zeyou Li for their assistance with HPLC experiments. We also thank Zemei Guo for the greenhouse assistance.

\section{Author details}

'Key Laboratory of Biology and Genetic Resources of Tropical Crops, Ministry of Agriculture and Rural Affairs \& Institute of Tropical Bioscience and Biotechnology, Chinese Academy of Tropical Agricultural Sciences, 571101 Haikou, China. ${ }^{2}$ Hainan Key Laboratory for Protection and Utilization of Tropical Bioresources \& Institute for Tropical Agricultural Resources, Chinese Academy of Tropical Agricultural Sciences, 571101 Haikou, China. ${ }^{3}$ College of Plant Protection, Hainan University, 570228 Haikou, China. ${ }^{4}$ College of Horticulture, Hainan University, 570228 Haikou, China. ${ }^{5}$ Hainan Key Laboratory of Tropical Microbe Resources, 571101 Haikou, China

\section{Author contributions}

W.S., P.Z., D.T., and P.Y. conceived and designed the research plan; D.T., G.Z., X.Y., H.W., and Y.L. performed the experiments; P.Y., H.C., G.Z., and X.L. analyzed the data; W.S. and P.Z. wrote the article.

\section{Conflict of interest}

The authors declare no competing interests.

Supplementary information The online version contains supplementary material available at https://doi.org/10.1038/s41438-021-00579-y.

Received: 2 February 2021 Revised: 13 April 2021 Accepted: 19 April 2021 Published online: 01 July 2021

\section{References}

1. da Silva, J. T. et al. Papaya (Carica papaya L.) biology and biotechnology. Tree Forestry Sci. Biotechnol. 1, 47-73 (2007).

2. Ming, R. et al. The draft genome of the transgenic tropical fruit tree papaya (Carica papaya Linnaeus). Nature 452, 991-996 (2008).

3. Nguyen, T. T., Shaw, P. N., Parat, M. O. \& Hewavitharana, A. K. Anticancer activity of Carica papaya: a review. Mol. Nutr. Food Res. 57, 153-164 (2013).

4. Saeed, F. et al. Nutritional and phyto-therapeutic potential of papaya (Carica papaya Linn.): an overview. Int. J. Food Prop. 17, 1637-1653 (2014).

5. Azarkan, M., El Moussaoui, A., van Wuytswinkel, D., Dehon, G. \& Looze, Y Fractionation and purification of the enzymes stored in the latex of Carica papaya. J. Chromatogr. B 790, 229-238 (2003).

6. Wei, F. \& Wing, R. A. A fruitful outcome to the papaya genome project. Genome Biol. 9, 227 (2008).

7. Gamboa-Tuz, S. D. et al. Transcriptomics and co-expression networks reveal tissue-specific responses and regulatory hubs under mild and severe drought in papaya (Carica papaya L.). Sci. Rep. 8, 14539 (2018).

8. Madronero, J. et al. Transcriptome analysis provides insights into the delayed sticky disease symptoms in Carica papaya. Plant Cell Rep. 37, 967-980 (2018).

9. Vargas-Mejía, P., Vega-Arreguín, J., Chávez-Calvillo, G., Ibarra-Laclette, E. \& SilvaRosales, L. Differential accumulation of innate- and adaptive-immune- response-derived transcripts during antagonism between Papaya ringspot virus and Papaya mosaic virus. Viruses 12, 230 (2020).

10. Zhu, $X$. et al. Transcriptomic analysis reveals key factors in fruit ripening and rubbery texture caused by 1-MCP in papaya. BMC Plant Biol. 19, 309 (2019).

11. Kung, Y. J. et al. Generation of hermaphrodite transgenic papaya lines with virus resistance via transformation of somatic embryos derived from adventitious roots of in vitro shoots. Transgenic Res. 19, 621-635 (2010).

12. Tecson Mendoza, E. M., A., C. L. \& Botella, J. R. Recent advances in the development of transgenic papaya technology. Biotechnol. Annu. Rev. 14, 423-462 (2008).

13. Fitch, M. M. M., Manshardt, R. M., Gonsalves, D. \& Slightom, J. L. Transgenic papaya plants from Agrobacterium-mediated transformation of somatic embryos. Plant Cell Rep. 12, 245-249 (1993).

14. Zhu, Y. J., Agbayani, R. \& Moore, P. H. Green fluorescent protein as a visual selection marker for papaya (Carica papaya L.) transformation. Plant Cell Rep. 22, 660-667 (2004).

15. Liu, Y., Schiff, M. \& Dinesh-Kumar, S. P. Virus-induced gene silencing in tomato. Plant J. 31, 777-786 (2002).

16. Robertson, D. VIGS vectors for gene silencing: many targets, many tools. Annu. Rev. Plant Biol. 55, 495-519 (2004).

17. Burch-Smith, T. M., Anderson, J. C., Martin, G. B. \& Dinesh-Kumar, S. P. Applications and advantages of virus-induced gene silencing for gene function studies in plants. Plant J. 39, 734-746 (2004)

18. Dommes, A. B., Gross, T., Herbert, D. B., Kivivirta, K. I. \& Becker, A. Virus-induced gene silencing: empowering genetics in non-model organisms. J. Exp. Bot. 70, 757-770 (2019).

19. Huang, C., Qian, Y., Li, Z. \& Zhou, X. Virus-induced gene silencing and its application in plant functional genomics. Sci. China-Life Sci. 55, 99-108 (2012).

20. Lange, M., Yellina, A.L., Orashakova, S. \& Becker, A. Virus-induced gene silencing (VIGS) in plants: an overview of target species and the virus-derived vector systems. in Virus-Induced Gene Silencing 975 (ed. Becker, A.) 1-14 (Humana Press, 2013)

21. Senthil-Kumar, M. \& Mysore, K. S. New dimensions for VIGS in plant functional genomics. Trends Plant Sci. 16, 656-665 (2011)

22. Tuo, D. et al. Complete genome sequence of an isolate of papaya leaf distortion mosaic virus from commercialized PRSV-resistant transgenic papaya in China. Acta Virol. 57, 452-455 (2013).

23. White, K. A. The polymerase slips and PIPO exists. EMBO Rep. 16, 885-6 (2015).

24. Tuo, D., Shen, W., Yan, P., Li, X. \& Zhou, P. Rapid construction of stable infectious full-length cDNA clone of papaya leaf distortion mosaic virus using In-Fusion cloning. Viruses 7, 6241-6250 (2015).

25. Tuo, D. et al. Generation of stable infectious clones of plant viruses by using Rhizobium radiobacter for both cloning and inoculation. Virology 510, 99-103 (2017).

26. Johansen, I. E. Intron insertion facilitates amplification of cloned virus CDNA in Escherichia coli while biological activity is reestablished after transcription in vivo. Proc. Natl Acad. Sci. USA 93, 12400-12405 (1996).

27. Gammelgård, E., Mohan, M. \& Valkonen, J. P. Potyvirus-induced gene silencing: the dynamic process of systemic silencing and silencing suppression. J. Gen. Virol. 88, 2337-2346 (2007)

28. Zhao, G. Y. et al. Profile of siRNAs derived from green fluorescent protein (GFP)-tagged Papaya leaf distortion mosaic virus in infected papaya plants. Virus Genes 54, 833-839 (2018).

29. Tuo, D. et al. A double mutation in the conserved motifs of the helper component protease of Papaya leaf distortion mosaic virus for the generation of a cross-protective attenuated strain. Phytopathology 110, 187-193 (2020).

30. Atreya, C. D., Atreya, P. L., Thornbury, D. W. \& Pirone, T. P. Site-directed mutations in the potyvirus HC-Pro gene affect helper component activity, virus accumulation, and symptom expression in infected tobacco plants. Virology 191, 106-111 (1992).

31. Gal-On, A. A point mutation in the FRNK motif of the potyvirus helper component-protease gene alters symptom expression in cucurbits and elicits protection against the severe homologous virus. Phytopathology 90, 467-473 (2000).

32. Lin, S. S., Wu, H. W., Jan, F. J., Hou, R. F. \& Yeh, S. D. Modifications of the helper component-protease of Zucchini yellow mosaic virus for generation of attenuated mutants for cross protection against severe infection. Phytopathology 97, 287-296 (2007).

33. Yan, P. et al. Nimble cloning: a simple, versatile, and efficient system for standardized molecular cloning. Front. Bioeng. Biotechnol. 7, 460 (2020). 
34. Sonderby, I. E., Geu-Flores, F. \& Halkier, B. A. Biosynthesis of glucosinolatesgene discovery and beyond. Trends Plant Sci. 15, 283-290 (2010).

35. Dong, Y., Burch-Smith, T. M., Liu, Y., Mamillapalli, P. \& Dinesh-Kumar, S. P. A ligation-independent cloning tobacco rattle virus vector for high-throughput virus-induced gene silencing identifies roles for NbMADS4-1 and -2 in floral development. Plant Physiol. 145, 1161-1170 (2007).

36. Folimonov, A. S., Folimonova, S. Y., Bar-Joseph, M. \& Dawson, W. O. A stable RNA virus-based vector for citrus trees. Virology 368, 205-216 (2007).

37. Rajamaki, M. L. et al. A novel insertion site inside the potyvirus P1 cistron allows expression of heterologous proteins and suggests some P1 functions. Virology 342, 88-101 (2005).

38. Tuo, D. C. et al. Two agroinfection-compatible fluorescent protein-tagged infectious cDNA clones of papaya leaf distortion mosaic virus facilitate the tracking of virus infection. Acta Virol. 62, 202-207 (2018).

39. Ghareeb, H., Laukamm, S. \& Lipka, V. COLORFUL-circuit: a platform for rapid multigene assembly, delivery, and expression in plants. Front. Plant Sci. 7, 246 (2016).

40. Donadio, L.C., Lederman, I.E., Roberto, S.R. \& Stucchi, E.S. Dwarfing-canopy and rootstock cultivars for fruit trees. Revista Brasileira de Fruticultura 41, e-997 (2019).

41. Ueguchi-Tanaka, M. et al. GIBBERELLIN INSENSITIVE DWARF1 encodes a soluble receptor for gibberellin. Nature 437, 693-698 (2005).

42. Ueguchi-Tanaka, M., Nakajima, M., Motoyuki, A. \& Matsuoka, M. Gibberellin receptor and its role in gibberellin signaling in plants. Annu. Rev. Plant Biol. $\mathbf{5 8}$ 183-198 (2007).

43. Liang, Y. C., Reid, M. S. \& Jiang, C. Z. Controlling plant architecture by manipulation of gibberellic acid signalling in petunia. Horticulture Res. 1, 14061 (2014).

44. Griffiths, J. et al. Genetic characterization and functional analysis of the GID1 gibberellin receptors in Arabidopsis. Plant Cell 18, 3399-3414 (2006).
45. Illouz-Eliaz, N. et al. Multiple gibberellin receptors contribute to phenotypic stability under changing environments. Plant Cell 31, 1506-1519 (2019).

46. Halkier, B. A. \& Gershenzon, J. Biology and biochemistry of glucosinolates. Annu. Rev. Plant Biol. 57, 303-333 (2006).

47. Petersen, A., Wang, C., Crocoll, C. \& Halkier, B. A. Biotechnological approaches in glucosinolate production. J. Integr. Plant Biol. 60, 1231-1248 (2018).

48. Reintanz, B. et al. bus, a bushy arabidopsis CYP79F1 knockout mutant with abolished synthesis of short-chain aliphatic glucosinolates. Plant Cell $\mathbf{1 3}$ 351-367 (2001).

49. Bennett, R. N., Kiddle, G. \& Wallsgrove, R. M. Biosynthesis of benzylglucosinolate, cyanogenic glucosides and phenylpropanoids in Carica papaya. Phytochemistry 45, 59-66 (1997).

50. Hansen, C. H. et al. CYP83B1 is the oxime-metabolizing enzyme in the glucosinolate pathway in Arabidopsis. J. Biol. Chem. 276, 24790-24796 (2001).

51. Smolen, G. \& Bender, J. Arabidopsis cytochrome P450 cyp83B1 mutations activate the tryptophan biosynthetic pathway. Genetics 160, 323-332 (2002).

52. Bak, S., Tax, F. E., Feldmann, K. A., Galbraith, D. W. \& Feyereisen, R. CYP83B1, a cytochrome $\mathrm{P} 450$ at the metabolic branch point in auxin and indole glucosinolate biosynthesis in Arabidopsis. Plant Cell 13, 101-111 (2001).

53. Maoka, T. \& Hataya, T. The complete nucleotide sequence and biotype variability of papaya leaf distortion mosaic virus. Phytopathology 95, 128-135 (2005).

54. Gadhave, K. R., Gautam, S., Rasmussen, D. A. \& Srinivasan, R. Aphid transmission of Potyvirus: the largest plant-infecting RNA virus genus. Viruses 12, 773 (2020).

55. Doheny-Adams, T., Redeker, K., Kittipol, V., Bancroft, I. \& Hartley, S. E. Development of an efficient glucosinolate extraction method. Plant Methods 13, 17 (2017). 